\title{
TECHNOLOGY OF AUTONOMOUS CARS
}

\author{
Himani Jain ${ }^{1}$, Divye Behl ${ }^{2}$, Shipra Saraswat ${ }^{3} \&$ Neetu Faujdar ${ }^{4}$
}

Abstract - In today's world time and life are the most valuable asset that humans have. The development of such autonomous cars will help mainly in saving time and preventing accidents which are mostly due to human errors. In this paper the authors have modelled the research direction towards the various technologies that the autonomous cars use to operate and locate themselves. The latest technologies aid these autonomous cars to be driven without any human help. This work provides the firsthand information to the readers who involves in the analysis and working of autonomous cars.

Keywords - Driving cars, Sensors, Technology

\section{INTRODUCTION}

An autonomous car is a vehicle that is capable of sensing its environment and navigating without human input [1]. It is basically the concept of developing such a car which would work without any human intermeddles and run on its own. Autonomous cars are also referred to as 'self driving cars' or 'driver less cars' as these cars do not need any driver to administrate them. These cars function on various sensors and location discoverers which are embedded into them. The sensors which are functional on these autonomous cars are some of the most advanced control systems. These cars can identify their surroundings and can also identify the roads, obstacles and different road signals. The control systems of autonomous cars are capable of analyzing sensory data to differentiate between various vehicles on the road which is very helpful in determining the path which the car takes and also gives very precise information about the cars surroundings. The history of making an autonomous car dates back to 1920's and 30's but the first functional autonomous car came out in 1980's. And since then many organizations and major automobile companies have been researching on autonomous cars [2-5].

Companies with major names in the field of automobile industry have always been working upon the concept of autonomous cars since day one. Now in 2016, some of the companies have already made prototypes and working models of autonomous cars which can run successfully on the roads. Autonomous cars now no longer remain a futuristic idea. Tesla, Ford, GM Motors, BMW, Audi are some of the companies which has given as estimate of year 2020 to 2021 as the year of launch of their self driving cars. The gradual increase of the estimated global installed base of cars with selfdriving features is also shown in the Fig 1 below. Google has already tested its prototype on the roads of California last summer. The time is not far when the next generation children would not believe that people used to drive cars manually.

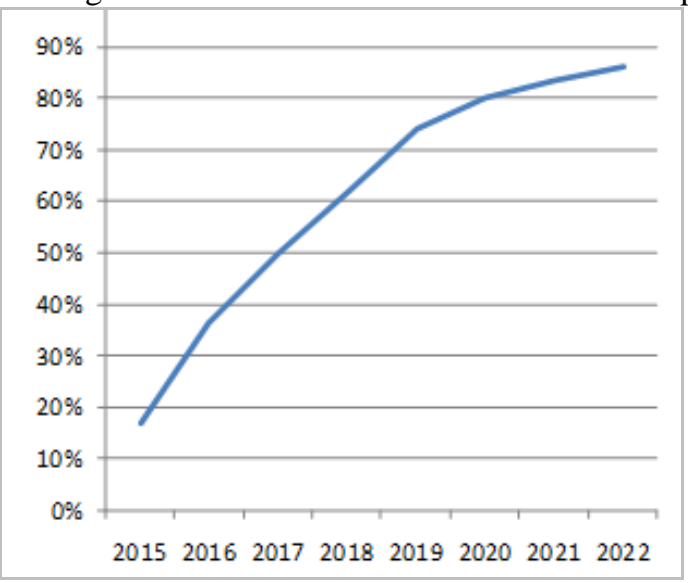

Fig. 1 Estimated Global Installed Base of Cars With Self-Driving Features

\section{RELATED WORK}

Autonomous cars are a very widely researched topic as these cars are the future of transportation arena. In this work, we focused on the technologies which will make the autonomous cars a success. In this section, we briefly review the related work by other researchers on autonomous cars.

In the book Autonomous Robot Vehicles (Ingemar J. Cox et al.), the authors presented the concept of autonomous vehicles. They have explained the way vehicles using autonomous aids work [6].

\footnotetext{
${ }^{1}$ Amity Institute of Engineering and Technology, Amity University Uttar Pradesh, Noida 201301, India

${ }^{2}$ Amity Institute of Engineering and Technology, Amity University Uttar Pradesh, Noida 201301, India

${ }^{3}$ Amity Institute of Engineering and Technology, Amity University Uttar Pradesh, Noida 201301, India

${ }^{4}$ Amity Institute of Engineering and Technology, Amity University Uttar Pradesh, Noida 201301, India
} 
The paper Online visual gyroscope for autonomous cars by Danial Kamran et al have presented the importance of gyroscope in navigating the autonomous robots. Gyroscope plays a major role in the working of autonomous cars as it is used to navigate [7].

Lin Li et al presented a new driver based model on human behaviour dynamics for autonomous cars, which allows the cars to move appropriately in accordance to the behavioural features of driver. It includes various properties, e.g. gender, age, driving experience, personality and emotions [8].

Every technology faces some issues. There are various issues related to autonomous cars too. This is proposed by Claudiu Pozna et al. in their paper titled Issues about Autonomous cars [9].

A planning method to obtain good quality paths for autonomous cars is presented by Domokos kiss et al in their paper. For non-holonomic system path planning among obstacles is still one of the most challenging problems in autonomous navigation [10]

\section{METHODOLOGY}

One thing that is always developing is the technology. Since cars came into business there has been endless improvement in their structure and the technical functionalities. Autonomous cars are different from the normal cars which need assistance of human inputs and so is the technology involved. Various varied sensor are at work when it is the sole responsibility of the car to drive safely by itself. These sensors are based on some of the most advanced researches and location detectors.

\subsection{Lidar}

The proposed autonomous cars will have a rotating roof top camera which is a laser range finder, Lidar. Lidar measures distance to a target by surveying method by illuminating it with a laser light. This camera by making a 3D image with its array of 64 laser beams will help the car see hazards along the way. As lidar operates with laser beams, the camera calculates the distance of any moving object from the car by the time the laser beam take to hit the object and come back. These impressive high intensity lasers can work within the range of $200 \mathrm{~m}$ to calculate distance and create images [7].

\subsection{Front Camera}

On the Windshield these cars will have a camera mounted on the windshield of the car to help the car detect objects in front of it. The objects will include the pedestrians and other vehicle users. The software built in the car would intelligently detect and record the traffic lights and the road signs.

\subsection{Radars}

There will be radars mounted on the front as well as the rear bumpers of these cars thus enabling them to be aware of the objects/vehicles in front and at the back of the car. This technology is similar to the cruise control in the present day cars. The radars present maintain a digital visual of the car ahead. The software of these cars are programmed to keep a distance of 4-5 seconds with the car ahead at all times. With this technology the car will automatically slow down or speed up according to the car in front of it. This technology will keep the passengers safe by avoiding unnecessary bumps and crashes.

\subsection{Aerial To Track The Location}

The rear of the car would have an aerial which would receive information about the exact location of the car with the help of GPS satellites. The car can locate itself with the sensors of the cars GPS inertial navigation unit. The GPS estimates can be inaccurate by some meters due to signal disturbance and other interferences from the atmosphere. To avoid such situations and minimise the risk the GPS data is compared to the data collected by the sensor maps previously from the same location.

\subsection{The Ultrasonic Sensor}

The ultrasonic sensor are mounted on the rear wheel of the car keeps the track of the cars movement and alerts about any obstacles in the rear. Today the advanced cars already have this technology in built in them. They use it for the reverse park assist which used this technology to help navigate the car into tight parking spots.

\subsection{The In Built Software}

The software of such cars are programmed to rightly interpret common road behaviour. The software is well designed to differentiate between a pedestrian, a cyclist or any other motorist signs. The car will automatically slow down to let the pedestrian pass to avoid any accidents. Also if the a cyclist is there the software is fed with various cuts the cyclist might make while on road and hence a pre fed information on the movement of such vehicles on roads help the autonomous cars to make a smart decision on road, thus avoiding any injury.

\subsection{Fed Features}

While the car is fed to slow down especially a four way intersections to allow other vehicles to go ahead, but in situations like if the other vehicle is not moving the car is programmed to advance ahead. 


\subsection{Altimeter}

An altimeter is an instrument used to measure the altitude of an object. Such altimeters are embedded into these cars to get a better idea to the location of the car.

\subsection{A tachymeter}

A tachymeter connected to the software of the car is used to calculate the speed based on travel time of the car.

\subsection{Gyroscopes}

Also the cars are fitted with gyroscopes which are used for maintain the orientation of the car. The fig 2 below shows the places where above mentioned sensors are placed.

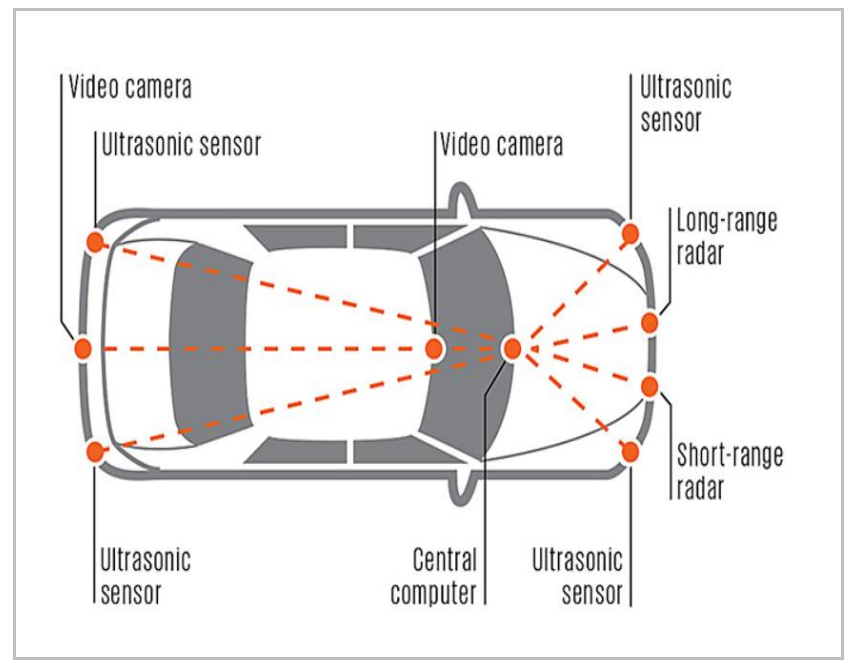

Fig. 2 Sensors in autonomous car

All the data provided by the sensors is gathered together and interpreted by the cars CPU to make intelligent and safe decisions about the movement of the car.

\section{COMPARING THE CURRENT AND AUTONOMOUS VEHICULAR SYSTEM ARCHITECTURE}

Comparing the current vehicular system architecture with the autonomous vehicular System architecture, with the help of the following diagrams we can come on the final point of comparison.

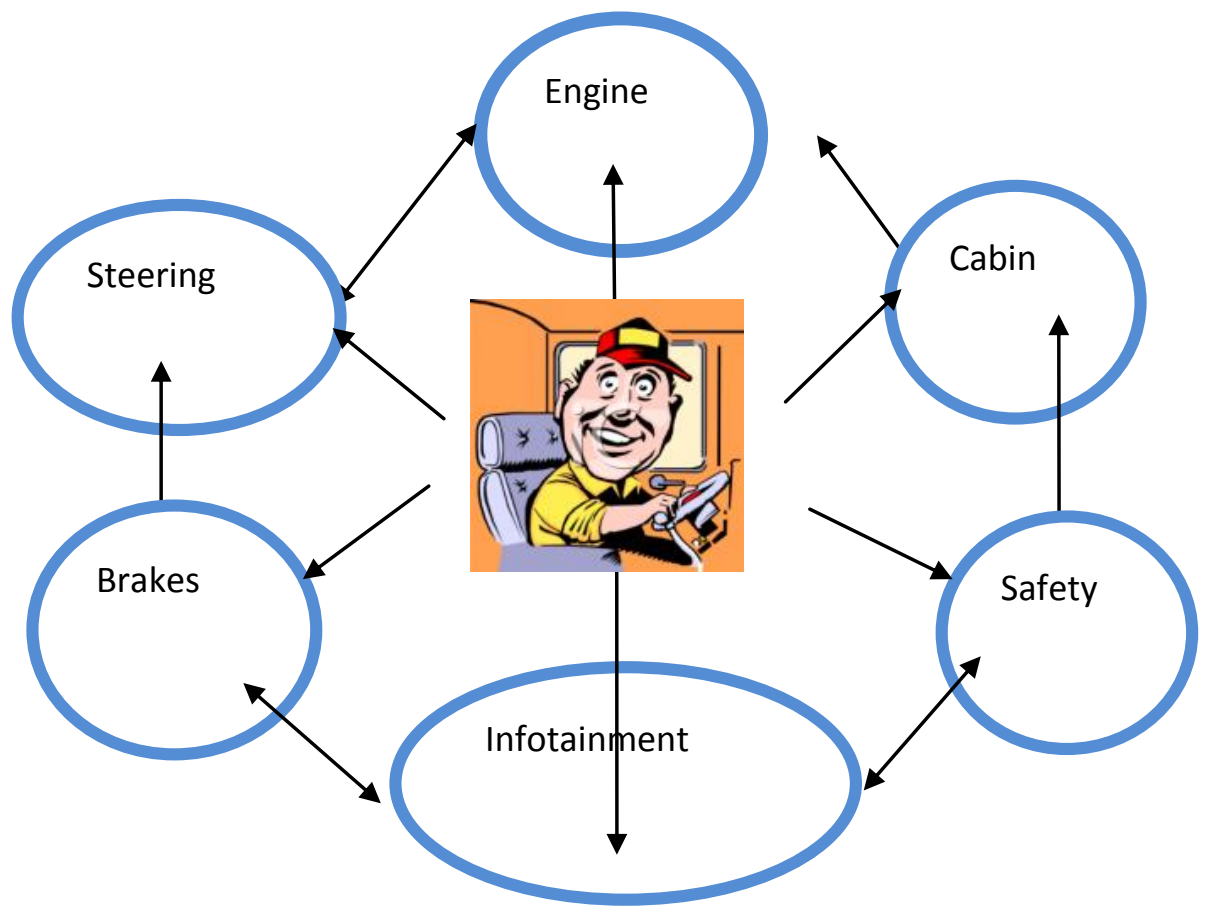

Fig. 3 Current Vehicular System's Architecture 


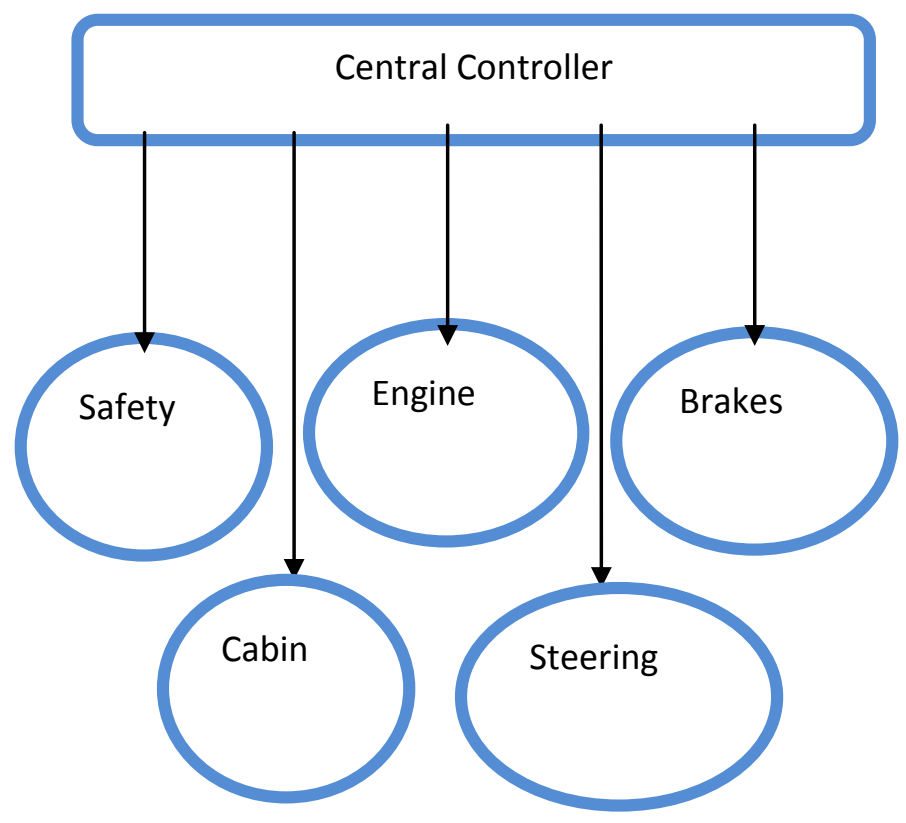

Fig. 4 Autonomous Vehicular System's Architecture

From the Fig $3 \&$ 4, we can clearly see that on one side in current vehicular System the driver is the controller of the System which is manual System. But on the other hand in second diagram it is a central Controller which is the operating System that controls the System. This means virtually very function of the car will now have a software component to it. In addition, every function within the car will now need to be supervised and controlled by the central computer, which runs an operating system similar to a personal computer, within with each of the different functions of the car reside. Unlike cars of today, with independent functions.

\section{BENEFITS OF AUTONOMOUS CARS}

\subsection{The Savings of using Autonomous Cars}

Now we have to measure the financial purposes of Self Sufficient vehicles. It has been estimated that Self Driven Vehicles can spare the US economy 1.3 trillion dollars annually reliably. We believe that these massive potential drift resources can animate the work of self driven vehicles. Now in this Block List Diagram we can see the savings that have been estimated in use due to these vehicles. Now this is a harsh survey that has been done. However it does not address the cost of executing self governing vehicles and theory preposition. The key that is giving motivation to the Autonomous vehicles is their ability to reduce the unpleasant social and economical effects of transportation structure. Here we have tried to figure out the aggregate potential cash related cost underpins that self driven vehicles address which is shown in Fig 5.

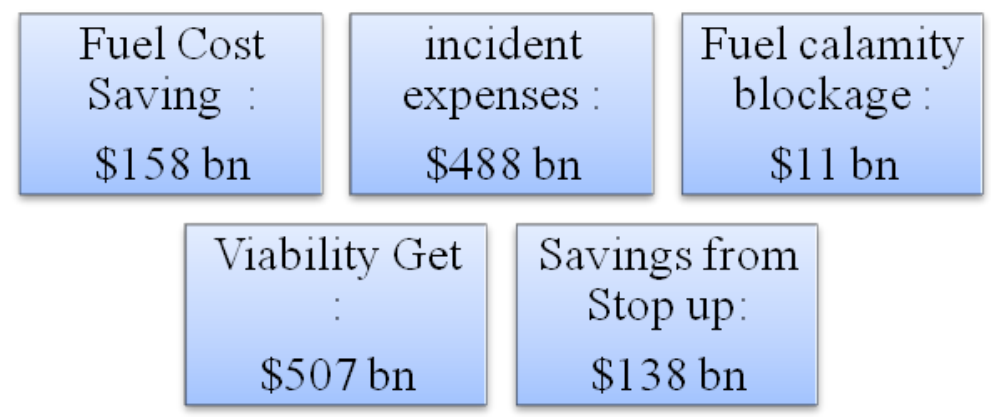

\subsection{Fewer Accidents}

Fig. 5 Savings from Autonomous Cars

The overheads that the society faces due the present vehicular system are the fatalities. In 2010 the World Flourishing Association (WHA) evaluated 1.2 million people losing their lives in light of vehicle collisions. One of the report by WHA examined that for all purposes a million adolescents are the victims of the after effect of the sudden injuries and the reason is auto-crash .mishaps are stunningly expensive. The far reaching costs join property hurt, medicinal cost, working environment cost, administrative cost, authentic cost and diminished individual fulfilment.

In all the cases $90 \%$ of the accidents are due to driver botch. Now expelling the driver from this condition will theoretically decrease the cost of such accidents and events by $90 \%$. This could save around $\$ 488$ dependably. This suggests right around $100 \%$ of the cars wherever ought to act typically palatable at all times to keep the one individual 
who is so far driving his auto himself from bringing on a disaster. As chose some time starting late, this will basically happen in the hopeful circumstance.

\subsection{Decreased Traffic}

We all know that the selfish behaviour of drivers is the most common reason that causes traffic jams and congestion. If drivers manage to give space to everyone and allow freely on the highways the traffic continues to move smoothly no matter how many cars are moving on the road. Self Driven Vehicles are programmed in such a way that they space out automatically on roads and thereby eliminating the problem of traffic jams.

\subsection{Enhanced Human Productivity}

Have you ever wondered that instead giving time to your car while driving you are giving time to yourself. Yes self driven cars will experience you that finish up a project, type a letter, manage your phone calls easily, read a book, watch a movie or obviously relax and enjoy your ride just like a passenger of a bus. Self driven cars would make you convert your time into productive time for whatever work you desire.

\section{SOFTWARE BUSINESS MODEL}

The average car today contains a reasonable amount of software about 5-10 million lines of code. The software in a car today typically regulates independent functions of a car, including drive-by-wire, traction management, active safety and infotainment. However, these systems act largely as independent silos today, with only a few handshakes or exchanges between components. The autonomous car of the future cannot work like this. All the systems in an autonomous car will need to be brought together within a central managing "brain" that can supervise and control almost every function of the car at all times. The level of system monitoring in an autonomous car is significantly higher than that of a regular car, given the fact that the main controlling factor of a regular car the driver is absent in an autonomous vehicle. The autonomous vehicle also cannot risk different sets of code written by different suppliers of each component. In effect, the central controller/operating system will be replacing the human driver as the primary operator of the vehicle.

\section{CONCLUSION}

This paper states that autonomous cars are the product of the latest technology which is growing every day. This study gives information on autonomous technology of production of cars that will revolutionize the transportation approach. The proposed paper will verify that our assumption of driving manual cars will be transmuted by a transportation system of fully autonomous vehicles in near future. In such an environment there would be less traffic, fewer accidents, less consumption of fuel and more efficient travelling.

\subsection{Time to reach there}

Now before we see the full introduction of the self driven cars in the market we need to resolve some issues outside the technology that is needed outside to get there. The issues may be short term or long term but these issues must be resolved for these vehicles to come in. We highlight the next three steps.

- Firstly the Government Support. We believe that the can play large role in the process to ramp up the self driven cars into the market including legislating the accommodation of self driven cars, there must be some special licenses for these vehicles in the early stage only helping the liability issue.

- Secondly building up consumer awareness. We know that it will be difficult for people to give up control on the steering wheel. Even after the introduction of cruise control many drivers are not in general use of this function because they cannot give up on the steering control. So the best way to introduce drivers to these self driven cars is conducting road shows at which they can be driven around small tracks in these cars at low speed so that they can become familiar with their use. We can also set up simulators so that the customers can try out the self driven cars in safe environment.

- Thirdly Resolving Legal Responsibility Issues. We believe that the legal responsibility needs to be carefully addressed soon because this the most frequently cited impediment to self driven cars. Now this is critical issue because early adoption of self driven cars might not be that that easy for both the government and the people.

\section{ACKNOWLEDGMENT}

The authors gratefully acknowledge the contributions of Shirpra Saraswat and Neetu Faujdar for their work on this document.

\section{REFERENCES}

[1] Jiajia Chen, Pan Zhao, Huawei Liang,Tao Mei, "Motion Planning for Autonomous Vehicle Based on Radial Basis Function Neural Network in Unstructured Environment”, sensors, pp. 17548-17566, doi:10.3390/s140917548, 2014.

[2] Garzn, M., Valente, J., Zapata, D., Barrientos, A., “An aerial-ground robotic system for navigation and obstacle mapping in large outdoor areas", Sensors, pp. 12471267, 2013.

[3] Budiharto, W., Purwanto, D., Jazidie, A., "A robust obstacle avoidance for service robot using Bayesian approach", International Journal of Adv. Robot System, 8, pp. 5260, 2011.

[4] Boumediene, S., Chourqaui, S., "Neural network navigation technique for unmanned vehicle” Trends Appl. Sci. Res, 9, pp. 246253, 2014.

[5] Chen, Q.Y., Sun, Z.P., Liu, D.X., Fang, Y.Q., Li, X.H., "Local path planning for an unmanned ground vehicle based on SVM", International Journal of Adv. Robot System, 9, doi:10.5772/54130, 2012. 
[6] E. Lindholm, J. Nickolls, S. F. Oberman, and J. Montrym, "Autonomous Robot Vehicles”, Springer Science \& Business Media, 2012.

[7] D. Kamran, M. Karimian, A. Nazemipour and M. Manzuri, "Online visual gyroscope for autonomous cars", 24th Iranian Conference on Electrical Engineering (ICEE), 2016.

[8] L. Li, Y. Liu, H. Oh, W. Deng and J. Wang, "Human dynamics based driver model for autonomous car", IET Intelligent Transport Systems, vol. 10 , no.8, pp. 545-554, 2016.

[9] C. Pozna and C. Antonya, "Issues about autonomous cars", IEEE 11th International Symposium on Applied Computational Intelligence and Informatics (SACI), 2016

[10] D. Kiss, G. Csorvasi and A. Nagy, "A Planning Method to Obtain Good Quality Paths for Autonomous Cars”, 4th Eastern European Regional Conference on the Engineering of Computer Based Systems, 2015. 